\title{
Interplay between quantum mechanical effects and a discrete trap position in ultra- scaled FinFETs
}

\author{
Vihar P. Georgiev ${ }^{\mathrm{a}}$, Salvatore M. Amoroso ${ }^{\mathrm{b}}$, Louis Gerrer ${ }^{\mathrm{a}}$, Fikru Adamu-Lema ${ }^{\mathrm{a}}$, Asen Asenov ${ }^{\mathrm{a}, \mathrm{b}}$ \\ ${ }^{a}$ Device Modelling Group, School of Engineering, University of Glasgow, Glasgow, G12 8LT, UK \\ ${ }^{b}$ Gold Standard Simulations Ltd, Glasgow G12 8QQ, UK \\ e-mail:vihar.georgiev@glasgow.ac.uk
}

\begin{abstract}
In this work we establish a link between positions of a single discrete charge trapped in an oxide interface and between the performance of ultra-scaled FinFET transistors. The charge trapped in the oxide induces gate voltage shift $\left(\Delta \mathbf{V}_{\mathrm{G}}\right)$. This $\Delta \mathbf{V}_{\mathrm{G}}$ is presented as a function of the device geometry for two regimes of conduction - from a sub-threshold to an ON-state. For specific trap positions in the oxide, we show that the trap impact decreases with scaling down of the FinFET size and of the applied gate voltage. We also compare the Drift-Diffusion (DD) calculations with the Non Equilibrium Green Functions (NEGF) simulations in order to investigate the importance of quantum charge confinement in transport and of reliability resilience in ultra-scaled non-planar transistors, such as FinFETs.
\end{abstract}

\section{INTRODUCTION}

$\mathrm{U}$ ltra-scale non-planar multi-gate transistors have become dominant architectures for the sub-22-nm technology [1]. However, even though the multi-gate transistors provide superior options for scalability, they also suffer from negative effects of various sources of variability [2-4]. For example, interface traps represent a non-negligible source of variability in ultra-scaled low-doped channel devices, such as FinFETs [4-6]. Therefore, in order to fully understand the nano-scaled FinFET performance it is essential to establish a correlation between the positions of the oxide-trapped charges in the presence of a strongly confined quantum effect and the device performance. It should be noted that the fin shape and the dimensions of the simulated FinFETs require quantum mechanical treatment of the electron transport [7]. For this reason in this paper we compare the threshold voltage shift amplitude $\left(\Delta \mathrm{V}_{\mathrm{G}}\right)$ obtained from the $\mathrm{DD}$ and NEGF computational results. We also study the impact of the device scaling on the gate voltage shifts associated with a single trapped charge and we investigate the influence of specific positions of the single trapped charge in the gate oxide on the FinFETs reliability performance.

\section{Methodology}

All simulations are carried out by a means of the DD module (including density-gradient quantum corrections) and the mode space NEGF approach module of the GSS atomistic

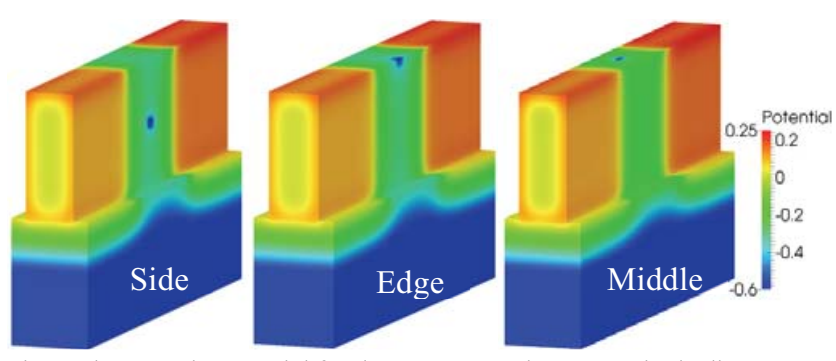

Fig. 1 Electrostatic potential for the $\mathrm{L}_{\mathrm{G}}=15 \mathrm{~nm}$ FinFET. A single discrete trap is located on the side (left) of the vertical oxide, at the edge of the fin

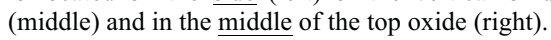

TABLE I

GEOMETRICAL DIMENSIONS AND MAIN CHARACTERISTICS FOR ALL FINFETS STUDIED IN THIS WORK.

\begin{tabular}{ccc}
\hline \hline $\begin{array}{c}\text { Gate Length }(\mathrm{L}) \\
{[\mathrm{nm}]}\end{array}$ & $\begin{array}{c}\text { Fin Width }\left(\mathrm{W}_{\text {FIN }}\right) \\
{[\mathrm{nm}]}\end{array}$ & $\begin{array}{c}\text { Fin Height }\left(\mathrm{H}_{\text {FIN }}\right) \\
{[\mathrm{nm}]}\end{array}$ \\
\hline 5 & 2 & 15 \\
10 & 4 & 15 \\
15 & 6 & 15
\end{tabular}

simulator GARAND [8, 9]. For the purpose of this work, we assume ballistic transport without scattering and absence of statistical variability. The ratio between the fin width and channel length is kept constant (see Table I) when scaling down the devices in order to maintain electrostatic integrity. Due to a single trapped charge located in the oxide, the gate voltage shifts $\left(\Delta \mathrm{V}_{\mathrm{G}}\right)$. This shift is evaluated by the transistor transfer characteristics in the sub-threshold regime $\left(\mathrm{V}_{\mathrm{G}}=0.05 \mathrm{~V}\right)$ and in the $\mathrm{ON}$-state regime $\left(\mathrm{V}_{\mathrm{G}}=0.6 \mathrm{~V}\right)$ at low drain biases $\left(\mathrm{VD}_{\mathrm{D}}=0.05 \mathrm{~V}\right)$. The effective oxide thickness is 0.8 $\mathrm{nm}$. The doping concentration in the source/drain region is $1 \mathrm{e} 20\left[\mathrm{~cm}^{-3}\right]$ and in the channel it is $1 \mathrm{e} 14\left[\mathrm{~cm}^{-3}\right]$. We consider only four of the six lowest valleys of the Si conduction band [9]. The effective masses correspond to their bulk values for $<100>$ crystal orientation in $\mathrm{Si}: m_{l}=0.916^{*} m_{0}$ and $m_{t}=0.19 * m_{0}$. It is important to point out that the effective masses do not scale with the device size in our calculations. This might be not sufficient for devices with severe charge confinement [10]. Further investigation is required and it is currently being undertaken. 


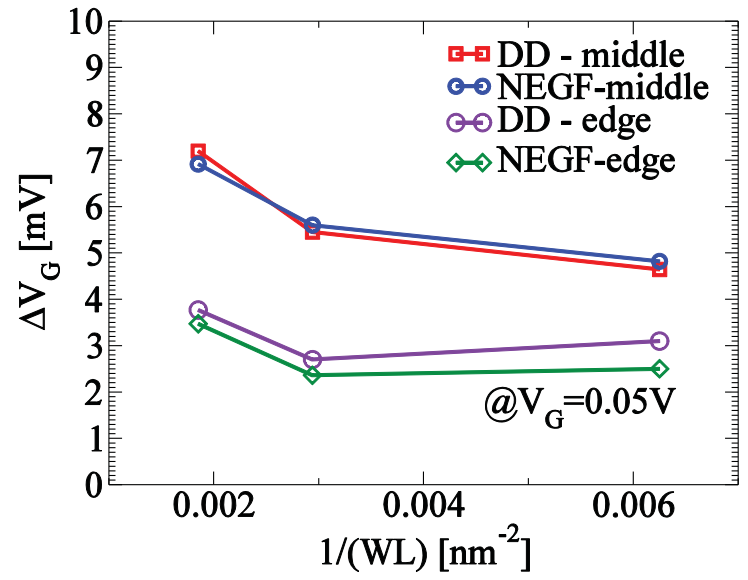

Fig. 2 Gate voltage shift due to a single discrete trapped charge at the edge of the fin and in the middle of the top oxide as a function of the FinFET active area size obtained by the DD and NEGF simulations with a current reading criterion in the sub-threshold region (100nA). Note, here $W=W_{F I N}+2 \times H_{F I N}$.

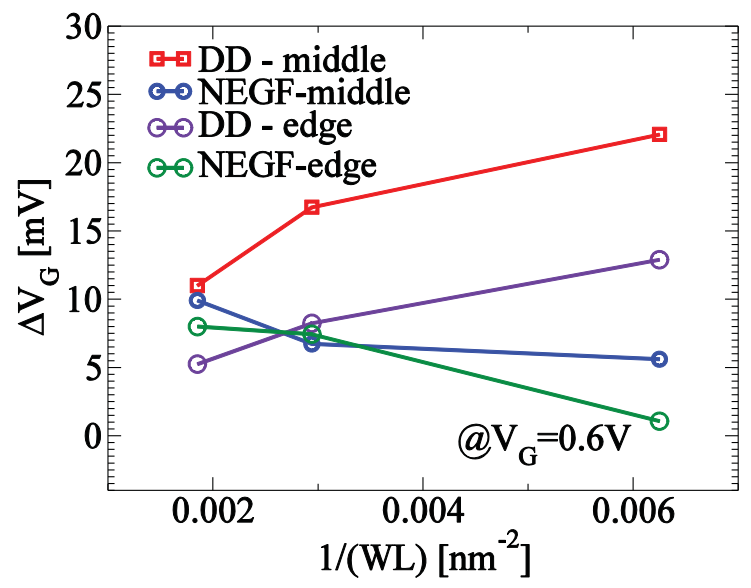

Fig. 3 Gate voltage shift due to a single discrete trapped charge at the edge of the fin and in the middle of the top oxide as a function of the FinFET active area size obtained by the DD and NEGF simulations with a current reading criterion in the $O N$-current region $(71 \mu \mathrm{A})$.

\section{RESULTS}

Fig. 1 shows the electrostatic potential for a device with the gate length of $15 \mathrm{~nm}$ and the fin width of $6 \mathrm{~nm}$. A fingerprint of a single trap located in the middle of the top oxide, at the edge of the top oxide and in the middle of the side oxide (Fig.1) is clearly visible.

Fig.2, Fig.3, Fig.4 and Fig.5 reveal the threshold voltage shift amplitude $\left(\Delta \mathrm{V}_{\mathrm{G}}\right)$ obtained for a current reading of $100 \mathrm{nA}$ and $71 \mu \mathrm{A}$ as a function of scaling. $\Delta \mathrm{V}_{\mathrm{G}}$ is obtained as the change of the gate voltage, allowing the same drain current for the empty (neutral) and the filled (negatively charged) trap.

Fig. 2 and Fig. 3 present the $\Delta \mathrm{V}_{\mathrm{G}}$ results at current reading of $100 \mathrm{nA}$ and $71 \mu \mathrm{A}$, respectively, for two types of devices - one with the edge and another one with the middle trap position. Fig. 2 shows that both transistors have a similar performance, with a surprising 'inverse-scaling' behaviour. Indeed, the impact of the trap located on top oxide of the fin increases when the fin size increases. This behaviour is opposite to the trend expected in conventional planar MOSTEFs $[11,12]$.

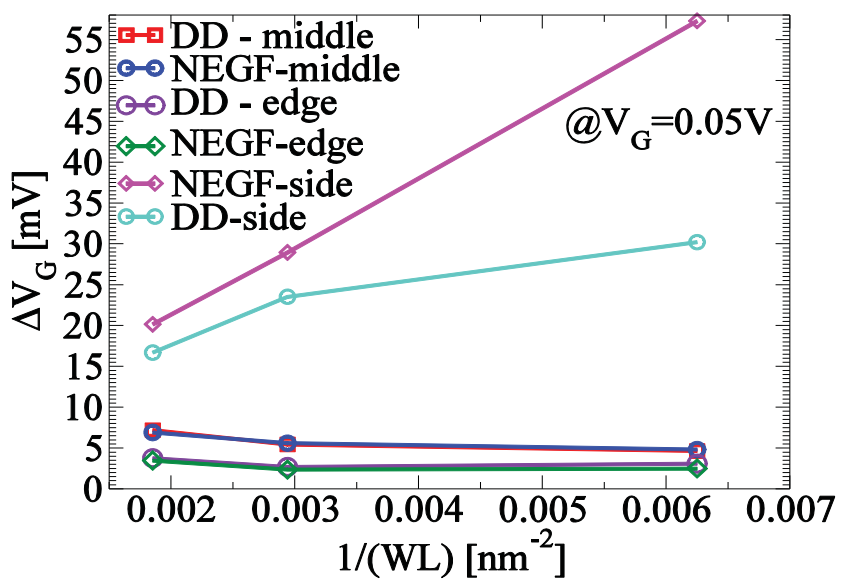

Fig. 4 Gate voltage shift due to a single discrete trapped charge located on the side of the vertical oxide, at the edge of the fin and in the middle of the top oxide as a function of the FinFET active area size obtained by the DD and NEGF simulations with a current reading criterion in the sub-threshold region (100nA).

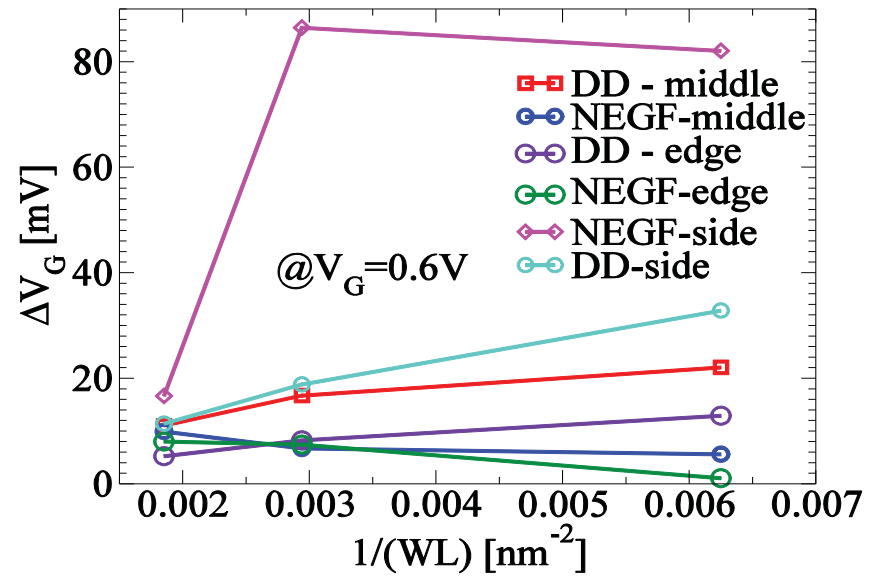

Fig. 5 Gate voltage shift due to a single discrete trapped charge located on the side of the vertical oxide, at the edge of the fin and in the middle of the top oxide as a function of the FinFET active area size obtained by the DD and NEGF simulations with a current reading criterion in the ON-current region $(71 \mu A)$.

Moreover, Fig. 3 shows the scaling of $\Delta \mathrm{V}_{\mathrm{G}}$ for the same two types of devices in the ON-state regime based on the DD and NEGF simulations. In this case the DD simulations show results that are consistent with a planar bulk transistor. Also, the NEGF calculations reveal lower $\Delta \mathrm{V}_{\mathrm{G}}$ values with a trend similar to the behaviour from Fig.2. Additionally, $\Delta \mathrm{V}_{\mathrm{G}}$ increases when moving from the sub-threshold to the ON-state region, in contrast to what is confirmed by conventional planar MOSFETs. It is important to point out that the DD and NEGF results confirm a positive trend between the $\Delta \mathrm{V}_{\mathrm{G}}$ value and the gate drive voltage.

However, an expected scaling trend is obtained for the transistor with the side trap position, which is shown in Fig.4. In Fig. 4 we also observe discrepancies in the $\Delta \mathrm{V}_{\mathrm{G}}$ trends between devices with traps on the top oxide and the device with the charged trapped on the side oxide. Also, for the side device the DD and NEGF results reveal a similar behaviour where both types of calculations reveal the expected scaling trends. 

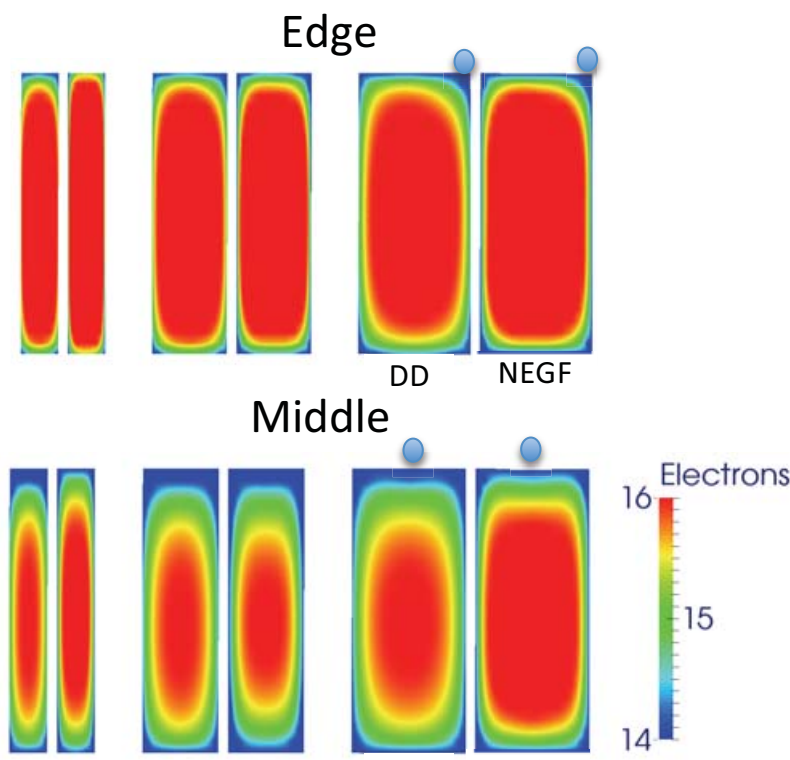

Side
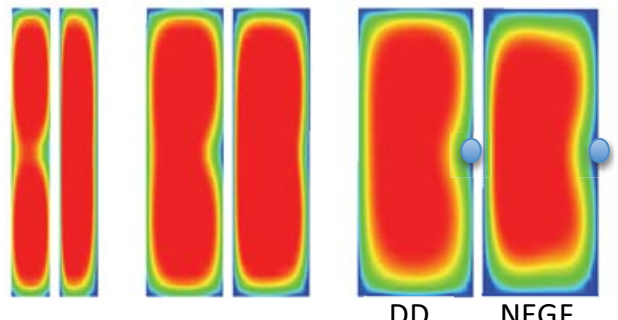

Fig. $62 \mathrm{D}$ cross-sections (at the center of the channel) of the simulate electron density at the threshold voltage conditions for a current criterion in the OFF-state regime $\left(V_{G}=0.05 \mathrm{~V}\right)$. The left (right) of each couple of the FinFET cross-sections represents the DD (NEGF) results. Top: FinFETs with the trap in the edge (top right corner); Middle: Transistors with the trap in the
middle of the top oxide; Bottom: Transistors with the trap at the side of the middle of the top oxide; Bottom: Transistor
fin (in the middle of the vertical right oxide).

Fig.5 shows the $\Delta \mathrm{V}_{\mathrm{G}}$ values and trends for all devices discussed in this work in the ON-state regime. It is clear from this figure that the DD calculations show the expected scaling behaviour. The NEGF curve for the side device shows behaviour that is close to the DD trend.

In order to understand the $\Delta \mathrm{V}_{\mathrm{G}}$ trends observed in the trigate architecture, in Fig.6 and Fig.7 we report the simulated $2 \mathrm{D}$ charge density at $\mathrm{V}_{\mathrm{G}}=0.05 \mathrm{~V}$ and $\mathrm{V}_{\mathrm{G}}=0.60 \mathrm{~V}$ correspondingly. Fig.6 shows that the channel inversion occurs deeply inside the bulk in the OFF-state regime and the profile of the charge distribution is similar for both the DD and NEGF simulations. As a result, the $\Delta \mathrm{V}_{\mathrm{G}}$ results reported in Fig. 2 and Fig. 4 have a similar trend with close values.

However, Fig. 7 reveals a different 2D charge distribution profile obtained with the NEGF and DD simulation methodology. From this figure we can draw the following important conclusions. Firstly, in all devices, the charge distribution moves closer to the oxide interface in the ON-state regime. This behaviour explains why the trap impact increases with increasing of $\mathrm{V}_{\mathrm{G}}$ (a common feature for the $\mathrm{DD}$ and NEGF simulations). It should be noted that this behaviour cannot be observed in the conventional planar MOSFET
Edge
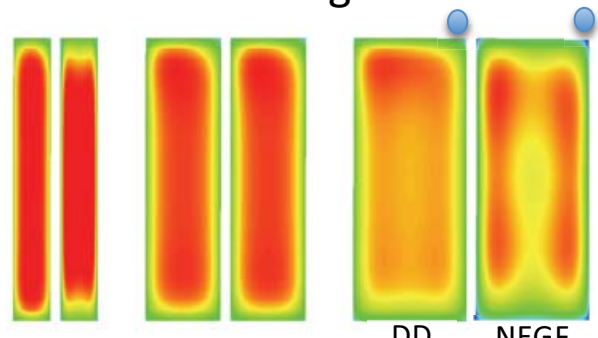

Middle
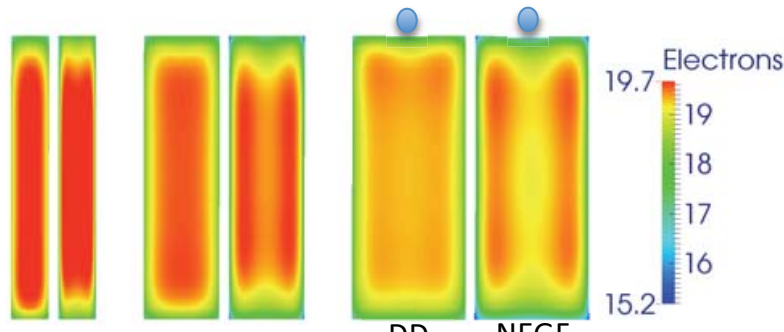

Side
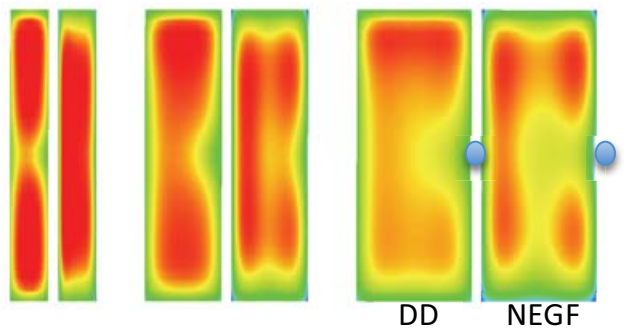

Fig. $72 \mathrm{D}$ cross-sections (at the center of the channel) of the simulated electron density at the threshold voltage conditions for a current criterion in the $O N$-state regime $\left(V_{G}=0.60 \mathrm{~V}\right)$. The left (right) of each couple of the FinFET cross-sections represents the DD (NEGF) results. Top: FinFETs with the trap in the edge (top right corner); Middle: Transistors with the trap in the middle of the top oxide; Bottom: Transistors with the trap at the side of the fin (in the middle of the vertical right oxide).

where the inversion charge centroid is always close to the oxide interface and barely modulated by the gate voltage conditions.

Secondly, the DD and NEGF charge distributions differ in the ON-state regime: while the DD charge moves uniformly towards the fin top interface, the NEGF charge follows a similar trend but it maintains two distinct peaks close to the lateral fin sidewalls. More importantly, it is evident that the DD charge distribution is mainly localised at the top interface of the fin, while the NEGF solution of the charge shows that it is predominately positioned at the vertical sidewalls of the fin. Also, the single trap charge in the oxide breaks the symmetry of the $2 \mathrm{D}$ charge distribution, which is visible at the $2 \mathrm{D}$ charge density plots for each device.

Additionally, it is important to point out that the device with the side trap shows the expected scaling behaviour at $\mathrm{V}_{\mathrm{G}}=0.05 \mathrm{~V}$ and $\mathrm{V}_{\mathrm{G}}=0.6 \mathrm{~V}$ (see Fig.4 and Fig.5). The main reason being that the charge inversion layer moves from the bulk body to the surface of the lateral gates. Moreover, the distribution of the charge along the length of the side gate is uniform and the profile is similar to what is observed in the planar MOSFETs. 


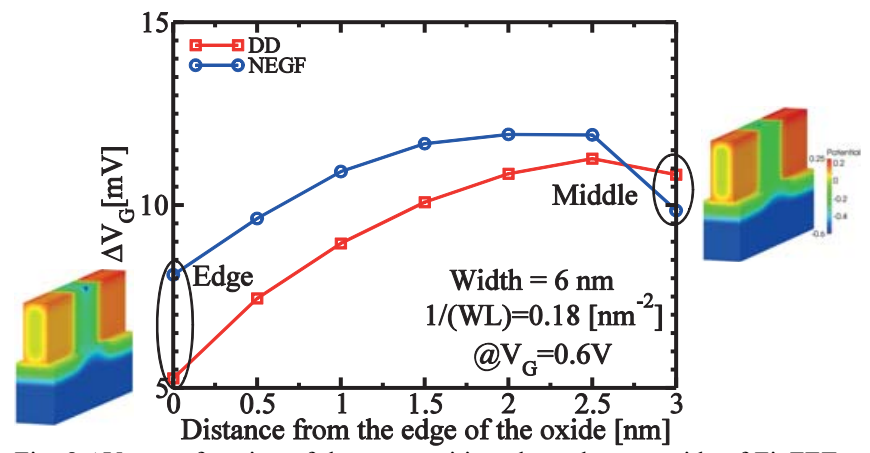

Fig. $8 \Delta \mathrm{V}_{\mathrm{G}}$ as a function of the trap position along the top oxide of FinFETs. The trap is moved from the edge of the fin to the middle of the top oxide.

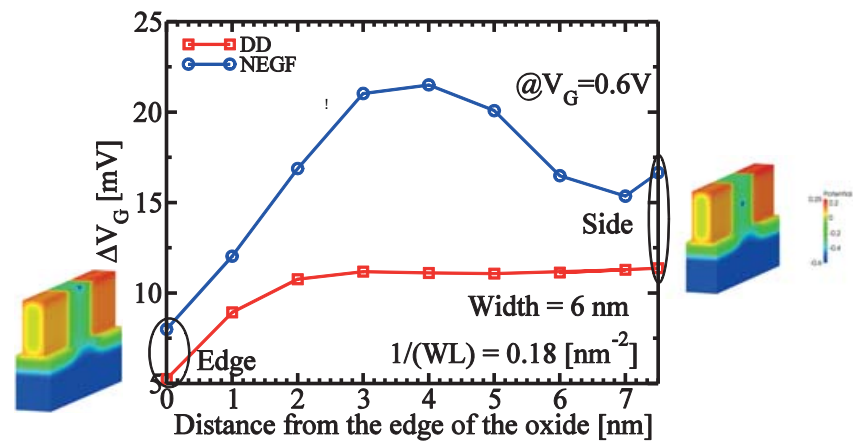

Fig. $9 \Delta \mathrm{V}_{\mathrm{G}}$ as a function of the trap position along the side oxide of FinFETs The trap is moved from the edge of the fin to the middle of the side oxide.

In order to clarify the link between the $2 \mathrm{D}$ charge distribution and $\Delta \mathrm{V}_{\mathrm{G}}$ we calculate sensitivity maps, which are shown in Fig.8 and Fig.9. Fig.8 presents the sensitivity map where the trap is moved with a step of $0.5 \mathrm{~nm}$ from the edge of the fin to the middle of the top oxide. In Fig.9 the trap is moved from the edge of the fin to the centre of the side oxide with a step of $1 \mathrm{~nm}$.

The results presented in Fig. 8 and Fig. 9 can be summarised in the following way. Both the DD and NEFG simulations show a specific behaviour. For example, in Fig.8, when the trap moves from the middle to the edge of the device, both the DD and NEGF calculations show a constant increase of the values of $\Delta \mathrm{V}_{\mathrm{G}}$. Also, values for $\Delta \mathrm{V}_{\mathrm{G}}$ obtained with the NEGF method are higher than for the DD calculations in most cases. The only exception is the middle device where the NEGF calculations give a number lower than the DD simulations. The reason for this is that in the NEGF case most of the charge inversion happens close to the corners of the device. Hence, the trap placed there in the NEGF simulations has a smaller impact on $\Delta \mathrm{V}_{\mathrm{G}}$.

Moreover, in Fig.9 the difference between the NEGF and DD results is even more pronounced. Moving a trap from the edge of the device to the side reveals a different behaviour for the DD and NEGF calculations. The impact of $\Delta \mathrm{V}_{\mathrm{G}}$ in the DD case is almost constant, while in the NEGF case the sensitivity curve shows an oscillating behaviour. The main reason being that the quantum effects lead to maintaining of two distinct peaks close to the lateral fin sidewalls. This is related to the complex 2D quantum charge distribution, especially at a high gate bias.

\section{CONCLUSIONS}

In this paper we presented calculations based on the $3 \mathrm{D}$ Drift-Diffusion and NEGF simulations, where we investigated the impact of the single discrete trapped charge on the performance of ultra-scaled nMOS FinFET transistors. The gate voltage shift was evaluated for three device sizes, several trap positions and for two regimes of conduction: subthreshold and $\mathrm{ON}$-state. We showed that in the sub-threshold region, due to the FinFET volume inversion, the trap located on the top oxide and the edge can show an 'inverse-scaling' behaviour contrary to what is expected in the conventional planar MOSFETs. However, the trap located on the side oxide of the FinFETs shows the expected gate voltage shift behaviour. More importantly, we showed that the quantum effects in charge distribution play an important role in evaluating the reliability issues in ultra-scaled devices.

\section{ACKNOWLEDGMENT}

The authors acknowledge the financial support provided by the European Commission through the FP7 grant agreement 261868 MORDRED.

\section{REFERENCES}

[1] D. A. Antoniadis and A. Khakifirooz, "MOSFET performance scaling: Limitations and future options," in Electron Devices Meeting, 2008. IEDM 2008. IEEE International, 2008, pp. 1-4.

[2] D. Linten, G. Hellings, C. Shih-Hung, and G. Groeseneken, "ESD in FinFET technologies: Past learning and emerging challenges," in Reliability Physics Symposium (IRPS), 2013 IEEE International, 2013, pp. 2B.5.1-2B.5.8.

[3] H. Kawasaki, V. S. Basker, T. Yamashita, C. Lin, Y. Zhu, J. Faltermeier, et al., "Challenges and solutions of FinFET integration in an SRAM cell and a logic circuit for $22 \mathrm{~nm}$ node and beyond," in Electron Devices Meeting (IEDM), 2009 IEEE International, 2009, pp. $1-4$.

[4] M. Toledano-Luque, B. Kaczer, J. Franco, P. J. Roussel, M. Bina, T. Grasser, et al., "Degradation of time dependent variability due to interface state generation," in VLSI Technology (VLSIT), 2013 Symposium on, 2013, pp. T190-T191.

[5] S. M. Amoroso, L. Gerrer, M. Nedjalkov, R. Hussin, C. Alexander, and A. Asenov, "Modeling Carrier Mobility in Nano-MOSFETs in the Presence of Discrete Trapped Charges: Accuracy and Issues," Electron Devices, IEEE Transactions on, vol. 61, pp. 1292-1298, 2014.

[6] W. Xingsheng, A. R. Brown, C. Binjie, and A. Asenov, "Statistical variability and reliability in nanoscale FinFETs," in Electron Devices Meeting (IEDM), 2011 IEEE International, 2011, pp. 5.4.1-5.4.4.

[7] S. M. Amoroso, V. P. Georgiev, L. Gerrer, E. Towie, W. Xingsheng, C. Riddet, et al., "Inverse Scaling Trends for Charge-Trapping-Induced Degradation of FinFETs Performance," Electron Devices, IEEE Transactions on, vol. 61, pp. 4014-4018, 2014.

[8] www.goldstandardsimulations.com.

[9] A. Martinez, M. Aldegunde, N. Seoane, A. R. Brown, J. R. Barker, and A. Asenov, "Quantum-Transport Study on the Impact of Channel Length and Cross Sections on Variability Induced by Random Discrete Dopants in Narrow Gate-All-Around Silicon Nanowire Transistors," Electron Devices, IEEE Transactions on, vol. 58, pp. 2209-2217, 2011.

[10] K. Nehari, N. Cavassilas, J. L. Autran, M. Bescond, D. Munteanu, and M. Lannoo, "Influence of band structure on electron ballistic transport in silicon nanowire MOSFET's: An atomistic study," Solid-State Electronics, vol. 50, pp. 716-721, 4// 2006.

[11] B. Kaczer, J. Franco, M. Toledano-Luque, P. J. Roussel, M. F. Bukhori, A. Asenov, et al., "The relevance of deeply-scaled FET threshold voltage shifts for operation lifetimes," in Reliability Physics Symposium (IRPS), 2012 IEEE International, 2012, pp. 5A.2.1-5A.2.6.

[12] S. M. Amoroso, C. M. Compagnoni, A. Ghetti, L. Gerrer, A. S. Spinelli, A. L. Lacaita, et al., "Investigation of the RTN Distribution of Nanoscale MOS Devices From Subthreshold to On-State," Electron Device Letters, IEEE, vol. 34, pp. 683-685, 2013. 